\section{Case Report}

Journal of Epilepsy Research pISSN 2233-6249 / eISSN 2233-6257

Received February 11, 2015

Accepted March 14, 2015

Corresponding author: Bong Su Kang Department of Neurology, Korea University Anam Hospital, 73 Inchon-ro, Seongbuk-gu, Seoul 136-705, Korea

Tel. +82-2-920-6264

Fax. +82-2-926-5347

E-mail; sookb99@gmail.com

\title{
A Case of Hyperventilation Syndrome Mimicking Complex Partial Seizure: Usefulness of EEG Monitoring in Emergency Department
}

\author{
Bong Su Kang \\ Department of Neurology, Korea University Anam Hospital, Seoul, Korea
}

\begin{abstract}
Acute hyperventilation syndrome not only can be clinically misdiagnosed as epileptic seizures, but also complex partial seizures may involve hyperventilation as a part of aura. Although electrography (EEG) monitoring is one of the most important procedure to differentiate these conditions, it could not be widely used in emergency department. Variety forms of epileptic attack, mainly idiopathic generalized epilepsy, are provoked by voluntary hyperventilation. In contrast, it is not clear whether hyperventilation can activate the partial seizures. We reported a case of acute hyperventilation syndrome (HSV) mimicking first onset complex partial seizure, impending non-convulsive status epilepticus, which was diagnosed by EEG in the emergency department. The electrographic seizure was provoked again by voluntary hyperventilation after clinical improvement. (2015;5:20-22)
\end{abstract}

Key words: Hyperventilation syndrome, Electroencephalography, Complex partial seizure

\section{Introduction}

Acute hyperventilation syndrome (HVS) represents a relatively common presentation at emergency department. The neurologist is likely to encounter HVS in the context of diverse symptoms including dizziness, palpitation, paresthesia in the extremities or periorally, muscle spasm related to tetany, confusion or agitation and depersonalization sensations. ${ }^{1}$ Epileptic seizures may also involve hyperventilation as part of the aura. ${ }^{1}$ On the other hands, hyperventilation is also considered to be a traditional seizure provoking procedure, especially in idiopathic generalized epilepsy rather than in partial epilepsy. $^{2}$

Although electroencephalography (EEG) monitoring is one of the most important procedure to differential these conditions, EEG could not be widely used in emergency department. We report a case of acute HVS mimicking first onset complex partial seizure, impending non-convulsive status epilepticus, which was diagnosed by EEG in the emergency department. The EEG seizure was provoked again by voluntary hyperventilation after clinical improvement.

\section{Case}

A 61-year-old previously healthy woman presented to emergency department with altered consciousness starting from 30 minutes ago. Her symptom was developed after blunt head trauma during personal assault. The patient had no history of medical illness including epilepsy or seizure, nor was there any relevant family history. Her vital signs were within normal range except for a high respiration rate of over $30 / \mathrm{min}$. On neurologic examination, the patient was confused, and could not respond appropriately to external stimuli just making incomprehensive sounds repeatedly. Recurrent tremulous movements in jaw and both hands were observed. There were no other lateralizing or localizing signs on examination.

The arterial blood gas analysis showed respiratory alkalosis $(\mathrm{pH}$ 7.431, $\mathrm{pCO}_{2} 28.0 \mathrm{mmHg}, \mathrm{pO}_{2} 100.6 \mathrm{mmHg}, \mathrm{HCO}_{3} 18.8 \mathrm{mmol} / \mathrm{L}, \mathrm{O}_{2}$ saturation $98.1 \%)$. There were no abnormalities on complete blood counts, chemistry panel, urinalysis and electrocardiography. There was no obvious evidence of brain injury in CT (Fig. 1A). Since her consciousness was not recovered until 2 hours after visiting, she underwent continuous EEG monitoring at emergency department. EEG revealed recurrent rhythmic theta waves in the right temporal areas with secondary bilateral synchrony (Fig. 2A, B). Brain MRI including diffusion weighted image showed no remarkable lesion except multiple dots in white mater (Fig. 1B). With intravenous lorazepam (4 mg) followed by intravenous levetiracetam $(2,000 \mathrm{mg})$ treatment, she recovered without any complication 1 day after admission. Follow-up 

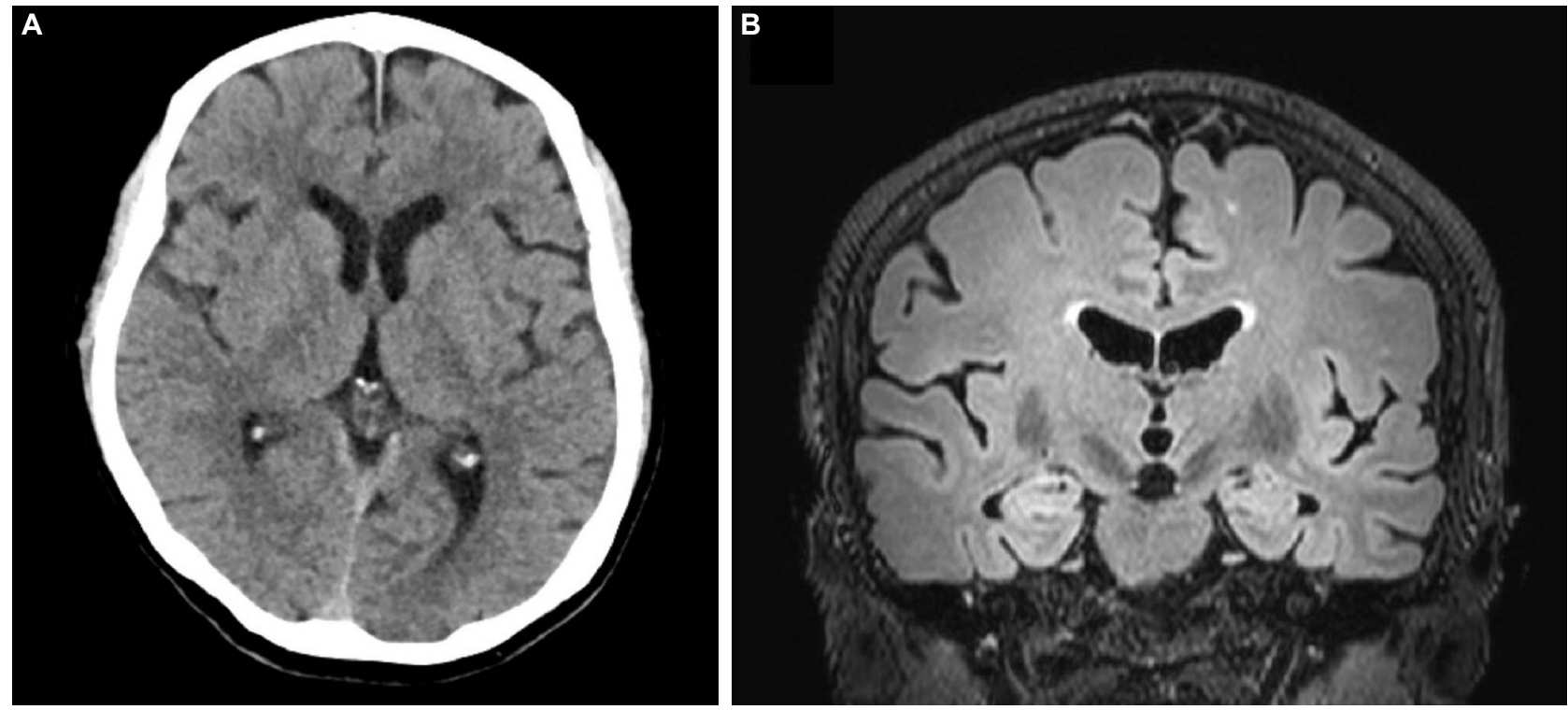

Figure 1. Brain CT. (A) reveals no abnormality and MRI (B) shows no remarkable abnormality, except white matter changes.
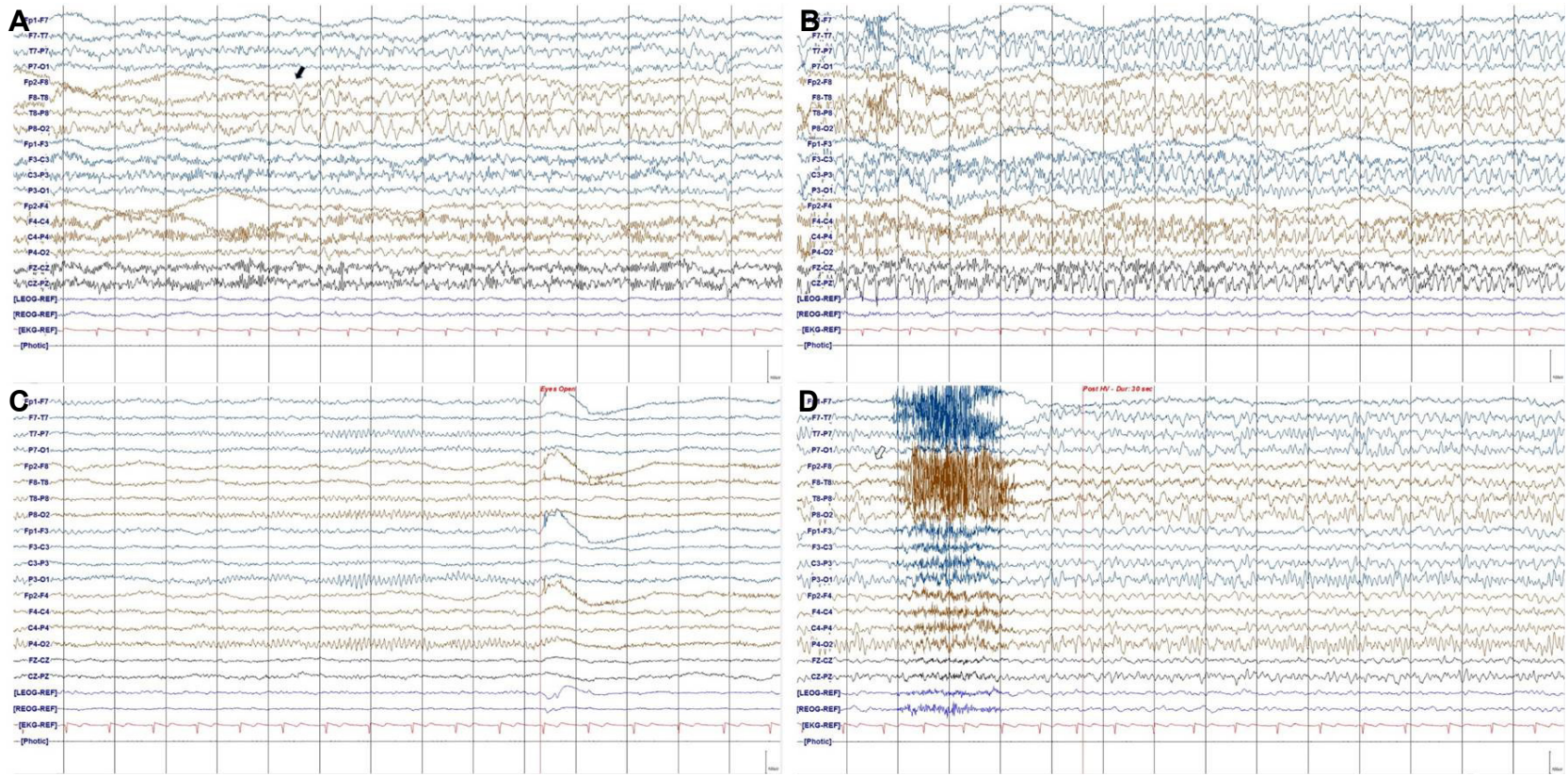

Figure 2. Continuous EEG monitoring shows (A) recurrent rhythmic theta wave activities in right temporal areas (black arrow) with (B) secondary bilateral synchrony. Followed EEG reveals (C) normal background activities and (D) provoked electrographic seizure after hyperventilation (white arrow).

EEG reveals normal background activities (Fig. 2C) but electrographic seizure was provoked at 30 seconds after hyperventilation ended (Fig. 2D).

\section{Discussion}

This is a case report of EEG based diagnosis of first onset recurrent complex partial seizures in emergency department which is provoked by hyperventilation. The clinical presentations which were confusion after minor head trauma associated with personal assault, sign of hyperventilation, respiratory alkalosis, normal brain image and no previous history of seizure or epilepsy could met a criteria of acute HVS. Acute HVS presents with obvious overbreathing and tachypnea and may have associated palpitation, dyspnea, dizziness, headache, muscle spasm related to tetany, paresthesis and syncope. ${ }^{3}$ Deja-vu or hallucination, which can make a referral to the epileptologist, have 
been rarely reported. ${ }^{4}$ HVS tends to be presented in aged between 15 and 55, occurs predominantly in women and sometimes have a history of psychosis. ${ }^{3}$ In the face of acute HVS, the clinician should exclude neurological or non-neurological organic cause including lesions in the pons or midbrain tegmentum, liver disease, cardiac disease and epileptic seizures. ${ }^{1}$ The neurologic manifestation of hyperventilation syndrome, such as "disorientation and twitching", can be clinically misdiagnosed as epileptic seizures. Occasionally, complex partial seizure (arising particularly from insular cortex) may involve hyperventilation as part of aura, although this is usually accompanied by automatism and other obvious signs of seizure. ${ }^{1}$

Hyperventilation is a well-established procedure for seizure provocation during performance of EEG, and the American EEG society (AEEGS, 2004), ${ }^{5}$ International League Against Epilepsy ${ }^{6}$ and National Institute for Health and Clinical Excellence ${ }^{7}$ all recommend that hyperventilation need to be performed as part of a standard EEG. It have been well known that many forms of epileptic seizures can be provoked by voluntary hyperventilation, especially effective in generalized epilepsies. ${ }^{2}$ A large-scaled study reported that 3-Hz generalized spike and waves were provoked by hyperventilation in $88 \%$ of 234 patients with childhood absence epilepsy. ${ }^{8}$ On the other hand, it is not clear whether hyperventilation can activate the partial seizures, such as our case. In recent published cases of seizures, the incidence of partial seizures during hyperventilation was more variable; with a range from none in 159 patients $^{9}$ up to $24.7 \%$ of 97 patients. $^{10}$

A rebuild-up of the high amplitude slow waves at 20-60 seconds after the hyperventilation ended is well known characteristic EEG pattern in pediatric moyamoya disease. ${ }^{11}$ It may be located in centrotemporal areas and be unilateral or bilateral. In this case, the electrographic seizure activities which was the evolving pattern of local slowing with bilateral synchronous generalized discharges could be distinguished from typical rebuild-up. 'In contrast, a recent large-scaled study reported that electrographic seizures were observed in 12 of 103 patients with adult moyamoya after cerebral revascularization. ${ }^{12}$ Since we did not evaluate the status of cerebral vasculatures, the possibility of moyamoya syndrome as an etiology of de novo partial onset seizures could not be ruled out.

EEG is one of the most important procedures for an accurate diagnosis of patients with altered mental status including HVS and epileptic seizures. Because treatment and prognosis of these conditions were different, accurate diagnosis should be crucial. However, EEG is not widely used in emergency department.

Our case suggests that EEG can be more useful modality for accu- rate diagnosis and treatment in cases with altered mentality and should be more considered in emergency department, even in a typical case with HVS.

\section{Acknowledgement}

There is no funding and financial or material support

\section{Conflict of Interest}

The authors have no financial conflict of interest

\section{References}

1. Engel J, Pedley TA. Epilepsy. A comprehensive textbook, 2nd ed. Baltimor: Williams \& wilkins 2008:2833-4.

2. Kane N, Grocott L, Kandler R, Lawrence S, Pang C.. Hyperventilation during electroencephalography: Safely and efficacy. Seizure 2014;23: 129-34.

3. Pincus JH. Disorders of conscious awareness. Hyperventilation syndrome. Br J Hosp Med 1978;19:312-3.

4. Allen $\mathrm{TE}$, Agus B. Hyperventilation leading to hallucinations. Am J Psychiatr 1968;125:632-7.

5. American EEG Society. Guideline One: minimum technical requirements for performing clinical electroencephalography. J Clin Neurophysio/ 1994; $11: 2-5$.

6. International League Against Epilepsy. Flink R, Pedersen $D$, Guekht $A B$, et al. Guidelines for the use of EEG methodology in the diagnosis of epilepsy. Acta Neurol Scand 2002;106:1-7

7. National Institute for Health and Clinical Excellence. The epilepsies: the diagnosis and management of the epilepsies in adults and children in primary and secondary care. 2012; NICE CG 137.

8. Dalby MA. Epilepsy and 3 per second spike and wave rhythms. A clinical, electrographic and prognostic analysis of 346 patients. Acta Neurol Scand 1969;45:1-83.

9. Abubakr A. Ifeayni I, Wambacq I. The efficacy of routine hyperventilation for seizure activation during prolonged video-electoencephalography monitoring. J Clin Neurosci 2010;17:1503-5.

10. Guaranha MSB, Garzon E, Buchpiquel CA, Tazima S, Yacubian EM, Sakamoto AC. Hyperventilation revisited: physiological effects and efficacy on focal seizure activation in the era of video-EEG monitoring. Epilepsia 2005:46:69-75.

11. Cho A, Chae $\mathrm{JH}, \mathrm{Kim} H M$, et al. Electroencephalography in pediatric moyamoya disease: reappraisal of clinical value. Childs Nerv Syst 2014; 30:449-59.

12. Frechette ES, Bell-Stephens TE, Steinberg GK, Fisher RS. Electrographic features of moyamoya in adults. Clin Neurophysio/ 2015;126: 481-5. 